PROCEEDINGS OF THE

AMERICAN MATHEMATICAL SOCIETY

Volume 134, Number 7, Pages 2139-2146

S 0002-9939(06)08200-1

Article electronically published on January 4, 2006

\title{
A CIRCLE PACKING MEASURABLE RIEMANN MAPPING THEOREM
}

\author{
G. BROCK WILLIAMS
}

(Communicated by Juha M. Heinonen)

\begin{abstract}
We prove a circle packing version of the Measurable Riemann Mapping Theorem in the spirit of Rodin and Sullivan's Circle Packing Riemann Mapping Theorem. We also construct circle packing maps of the plane onto itself with prescribed dilatation.
\end{abstract}

\section{INTRODUCTION}

Classically, the distortion of a quasiconformal map $f$ is described by its complex dilatation $\mu$, defined by the Beltrami equation

$$
\bar{\partial} f=\mu \partial f .
$$

Conversely, $f$ is quasiconformal only if it is the solution of (1.1) for some Beltrami differential $\mu \in L^{\infty},\|\mu\|_{\infty}<1$ [8, 14, 15]. The classical Measurable Riemann Mapping Theorem asserts that given a Beltrami differential $\mu$ on a simply connected domain $\Omega \subsetneq \mathbb{C}$, there is a corresponding quasiconformal map $f^{\mu}$ from $\Omega$ to the unit disc $\mathbb{D}$ having $\mu$ as its complex dilatation. If $f^{\mu}$ is normalized to send two points $z_{0}, z_{1} \in \Omega$ to 0 and the positive real axis, respectively, then $f^{\mu}$ is unique. The original Riemann Mapping Theorem follows from the special case $\mu=0$.

Circle packings have been used previously by Z.X. He [9] to solve Beltrami differential equations, but they appear indirectly. The distortion was produced by precomposition with a quasiconformal map. In this note, we prove a direct circle packing version of the Measurable Riemann Theorem in the spirit of Rodin and Sullivan's Circle Packing Riemann Mapping Theorem [17.

Rodin and Sullivan constructed circle packing maps from a simply connected region $\Omega \subsetneq \mathbb{C}$ to $\mathbb{D}$ by first filling $\Omega$ with a circle packing, then creating a packing in $\mathbb{D}$ with the same combinatorial structure. The resulting map is approximately conformal. We construct quasiconformal circle packing maps by altering the combinatorial structure of the of the first packing before creating the range packing in $\mathbb{D}$. By controlling the combinatorial distortion, we can precisely control the quasiconformal distortion between the two packings.

Received by the editors December 4, 2002 and, in revised form, February 10, 2005

2000 Mathematics Subject Classification. Primary 52C26, 30F60.

Key words and phrases. Circle packing, quasiconformal maps, Teichmüller theory.

The author gratefully acknowledges the support of the Texas Tech University Research Enhancement Fund.

(C)2006 American Mathematical Society Reverts to public domain 28 years from publication 
In Lehto and Virtanen's classic proof of the Measurable Riemann Mapping Theorem [15], quasiconformal maps are first constructed on small rectangles where the complex dilatation can be assumed to be constant. The rectangle maps are then glued together using conformal welding. Our approach also employs a circle packing version of conformal welding; however, we use welding to create the quasiconformal distortion itself rather than to piece together maps which are already quasiconformally distorted.

We begin with a discussion of circle packings and their deep connection to conformal maps in Section 2. In Section 3, we construct quasiconformally distorted circle packing maps of the plane as a prelude to our Circle Packing Measurable Riemann Mapping Theorem in Section 4

\section{Conformal Circle PaCKing Maps}

Definition 2.1. A circle packing $\mathcal{P}_{\mathcal{K}}$ for a triangulation $\mathcal{K}$ is a locally finite configuration of circles $\left\{\mathcal{C}_{v}\right\}$ indexed by the vertices of $\mathcal{K}$ with $\mathcal{C}_{v}$ tangent to $\mathcal{C}_{w}$ if the vertices $v$ and $w$ are joined by an edge in $\mathcal{K}$.

Notice that by connecting centers of circles with line segments, we can recover the original triangulation $\mathcal{K}$ from the packing. The region covered by these triangles form the carrier $|K|$ of the packing. A packing is univalent if none of its circles overlap.

If $\mathcal{K}$ is a simply connected triangulation, the existence of a univalent packing $\mathcal{P}$ for $\mathcal{K}$ was proven independently by Koebe [13, Andreev [1, and Thurston [16, 19]. If the triangulation is infinite, then $\mathcal{P}$ fills either the complex plane $\mathbb{C}$ or the unit disc $\mathbb{D}$ and is unique up to conformal automorphisms [3. If the triangulation is finite and planar, much more variety is possible; however, one can always find a packing which fills $\mathbb{D}$ and is unique up to conformal automorphisms.

Example 2.2. One useful infinite packing is the "ball bearing packing" named by Dubejko and Stephenson [7. The underlying triangulation is created from a lattice, and the original lattice structure is still apparent in the resulting packing. Consequently, the carrier of the packing can be decomposed into small squares. Moreover, there is a natural refinement of the triangulation and carrier created by replacing each square with four copies of the original. See Figure 1.

Notice that the large "lattice" circles (corresponding to vertices in the original lattice) are all the same size and the smaller "ball bearing" circles are all the same size. We have recently used deformations of this packing to show that every noncompact Riemann surface can be filled with a circle packing [23].

The intense interest in circle packings began when Thurston 20] conjectured and Rodin and Sullivan [17 proved a circle packing version of the Riemann Mapping Theorem. Let $\Omega \subsetneq$ be a simply connected region and let $z_{0}, z_{1} \in \Omega$. Let $\left\{\mathcal{P}_{n}\right\}$ be a sequence of circle packings in $\Omega$ whose carriers $\left|\mathcal{K}_{n}\right|$ converge to $\Omega$ and whose meshes converge to 0 . Suppose in each packing, two circles $\mathcal{C}_{0}$ and $\mathcal{C}_{1}$ are centered at $z_{0}$ and $z_{1}$, respectively. It follows from Koebe's, Andreev's, and Thurston's Theorem that each $\mathcal{P}_{n}$ can be repacked as a packing $\widetilde{\mathcal{P}}_{n}$ in the unit disc $\mathbb{D}$ with the circles corresponding to $\mathcal{C}_{0}$ and $\mathcal{C}_{1}$ centered at 0 and on the positive real axis, respectively. Since $\mathcal{P}_{n}$ and $\widetilde{\mathcal{P}}_{n}$ share the same underlying triangulation, there is a natural map $f_{n}$ between their carriers defined by sending centers of circles to centers of circles and extending linearly on the triangles. 


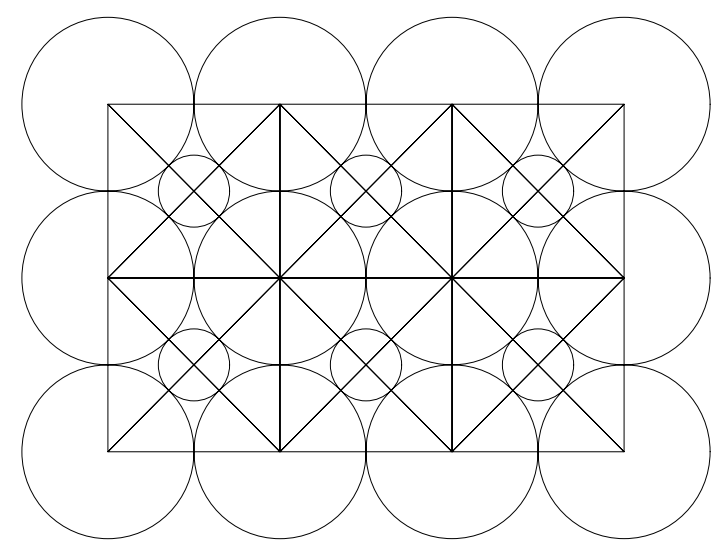

Figure 1. A portion of the "ball bearing" packing. The carrier has been drawn in to emphasize the lattice structure.

Circle Packing Riemann Mapping Theorem. The circle packing maps $f_{n}$ converge uniformly on compact subsets of $\Omega$ to the Riemann map $f: \Omega \rightarrow \mathbb{D}$ with $f\left(z_{0}\right)=0$ and $f\left(z_{1}\right)>0$.

Rodin and Sullivan's original proof applied only to packings in which each circle had six neighbors, but it was extended by Stephenson [18, and Rodin and He [10, with the most general version due to He and Schramm [12].

It is now a well-established folk theorem that circle packings strongly "want" to be conformal. For example, packings respond to the introduction of branch points [6], subdivision rules [5], and various surgeries [2, 22, 24] by approximating the conformal map being modeled.

As a result, the only hope for producing quasiconformal circle packing maps is to change the underlying triangulation. The combinatorial changes will be reflected in the geometry of the new packing, and the change in geometry will result in a quasiconformal deformation.

\section{QUASICONFORMAL CIRCLE PACKING MAPS}

3.1. Maps of the entire plane. At the heart of Rodin and Sullivan's proof is the uniqueness of normalized packings filling the complex plane. This is a circle packing analog of the fact that the only normalized univalent map of the plane to itself is the identity. Thus as a first step toward a circle packing Measurable Riemann Mapping Theorem, we construct circle packing maps converging to quasiconformal maps on the entire plane.

Consider a constant Beltrami differential $\mu_{0}$ on the entire plane. Unlike the general case, there is an obvious linear quasiconformal map

$$
f^{\mu_{0}}(z)=\frac{z+\mu_{0} \bar{z}}{1+\mu_{0}}
$$

with complex dilatation $\mu_{0}$ and fixing 0 and 1.

Let $\mathcal{P}$ be the infinite ball-bearing packing of Example 2.2 and $\mathcal{K}$ its triangulation. We may assume $\mathcal{P}$ is normalized so that two circles $\mathcal{C}_{0}$ and $\mathcal{C}_{1}$ are centered at 0 


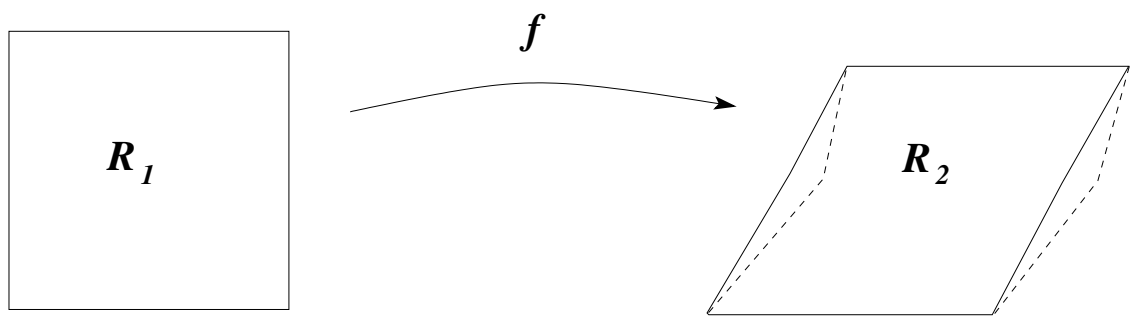

Figure 2. Fundamental quadrilaterals $R_{1}$ and $R_{2}$ which are not conformally equivalent. However, if $R_{2}$ is bent into another fundamental domain $\widetilde{R_{2}}$ (dashed), then there will exist a conformal map $f: R_{1} \rightarrow \widetilde{R_{2}}$.

and 1 , respectively. Recall that $\mathcal{P}$ is composed of many square pieces - four large "lattice" circles with a smaller "ball bearing" circle inside. By modifying these pieces, we will create a combinatorial distortion which reflects the quasiconformal distortion encoded by $\mu_{0}$.

3.2. Conformal welding. In earlier work with Roger Barnard [2, we described a deformation for circle packings on tori. Recall that every torus can be represented as the plane modulo a lattice. Thus deforming one torus into another means deforming the underlying lattice structure. The lattice produces a tiling of the plane by fundamental parallelograms. By cutting adjacent parallelograms apart and gluing them (unevenly) back together, we distort the lattice. This idea of uneven gluing is formalized in the notion of conformal welding [15, 8, 14, 4.

Definition 3.1. Let $\Omega^{+}$and $\Omega^{-}$be regions in $\mathbb{C}$ and $\varphi$ a homeomorphism between (portions of) their boundaries. Identify points which correspond under $\varphi$ to create a new region $\Omega$. We say $\Omega$ can be produced by conformally welding $\Omega^{+}$and $\Omega^{-}$ by $\varphi$ if there exist conformal maps $f^{+}: \Omega^{+} \rightarrow \Omega$ and $f^{-}: \Omega^{-} \rightarrow \Omega$ onto disjoint subsets of $\Omega$ whose boundary values satisfy the welding condition

$$
\left(f^{+}\right)^{-1} \circ f^{-}=\varphi \text {. }
$$

Suppose $T_{1}$ and $T_{2}$ are tori with conformally different underlying lattices. It follows from the work of Toki 21 that there exist welding homeomorphisms on each edge of each fundamental parallelogram in the lattice for $T_{1}$ which produce fundamental parallelograms which generate the lattice for $T_{2}$. The explicit homeomorphisms are presented in our earlier work with Roger Barnard [2]. For completeness sake, we briefly describe the construction here.

Since the lattices for $T_{1}$ and $T_{2}$ are conformally distinct, any fundamental parallelograms $R_{1}$ and $R_{2}$, for $T_{1}$ and $T_{2}$, respectively, must be conformally inequivalent. That is, there can exist no conformal map from $R_{1}$ onto $R_{2}$ which preserves the lattice vertices. However, $R_{2}$ can be "bent" into a region $\widetilde{R_{2}}$ which is conformally equivalent to $R_{1}$. That is, there will exist a conformal map $f: R_{1} \rightarrow \widetilde{R_{2}}$ which preserves the original vertices. See Figure 2

Since $R_{1}$ and $R_{2}$ generate the entire original lattices by translation, the map $f$ generates maps on every fundamental region in the lattice. For example, suppose $R_{1}^{+}$and $R_{1}^{-}$are fundamental parallelograms in the lattice for $T_{1}$ sharing a common edge $L$. Then there exist conformal maps $f^{+}$and $f^{-}$on $R_{1}^{+}$and $R_{1}^{-}$, respectively, 

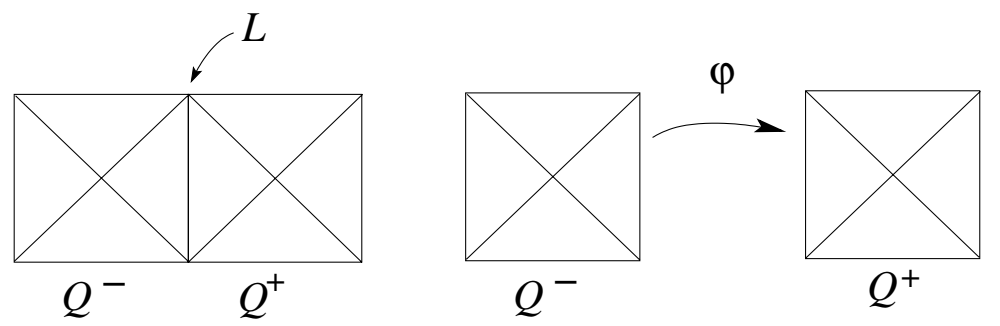

Figure 3. Two adjacent quadrilaterals $Q^{+}$and $Q^{-}$(left) with a homeomorphism $\varphi$ mapping their common edge $L$ onto itself (right).

onto adjacent regions in the lattice for $T_{2}$ which send lattice vertices to lattice vertices. If we define $\varphi: L \rightarrow L$ by

$$
\varphi(z)=\left(f^{+}\right)^{-1} \circ f^{-}(z),
$$

then our construction shows a conformal welding exists for $\varphi$. Repeating this welding throughout the lattice for $T_{1}$ will transform it into the lattice for $T_{2}$.

3.3. Welding packings. Returning to the idea of distorting the lattice formed by the triangulation $\mathcal{K}$ of the ball-bearing packing, if $Q^{+}$and $Q^{-}$are adjacent squares in $\mathcal{K}$, then we can treat their realizations $\left|Q^{+}\right|$and $\left|Q^{-}\right|$in the carrier of $\mathcal{P}$ as fundamental domains for a square torus and apply the above procedure. Let $L=\left|Q^{+}\right| \cap\left|Q^{-}\right|$be the common side of $Q^{+}$and $Q^{-}$and suppose $\varphi$ is the welding homeomorphism of $L$ onto itself. See Figure 3 ,

For our circle packing version, we need to create a new welded triangulation from $Q^{+}$and $Q^{-}$. Thus we will glue vertices on the side of $Q^{+}$to vertices on the side of $Q^{-}$. As it stands now, however, there are no vertices on the sides of $Q^{+}$and $Q^{-}$ which we can glue.

To remedy this situation, for each $m$, we let $\mathcal{K}_{m}$ be the $m^{\text {th }}$ refinement of $\mathcal{K}$ as described in Example 2.2, In the refined complex, $m$ vertices have been created on each side of $Q$ and $Q^{-}$. These vertices can be naturally embedded as equally spaced points on the sides of $\left|Q^{+}\right|$and $\left|Q^{-}\right|$. We then use $\varphi$ to determine how to glue the vertices on the side of $Q^{+}$to those on $Q^{-}$. The only difficulty lies in the fact that the image of a vertex on $Q^{+}$might lie between two vertices on $Q^{-}$. Thus we will need to take a common refinement of the sides, so that vertices will map to vertices.

Repeating this process on every square in $\mathcal{K}_{m}$, we create a welded triangulation $\widetilde{\mathcal{K}}_{m}$. It is easy to verify using He and Schramm's parabolicity criteria 11 that $\widetilde{\mathcal{K}}_{m}$ will have packing $\widetilde{\mathcal{P}}_{m}$ filling $\mathbb{C}$ which we can normalize so that the circles corresponding $\mathcal{C}_{0}$ and $\mathcal{C}_{1}$ in $\mathcal{P}$ are still centered at 0 and 1 , respectively.

Notice that our welded triangulation $\widetilde{\mathcal{K}}_{m}$ still contains the original vertices of each square in $\mathcal{K}$. Thus we can define a map $f_{m}:|\mathcal{K}| \rightarrow\left|\widetilde{\mathcal{K}}_{m}\right|$ by sending these vertices in $|\mathcal{K}|$ to the corresponding vertices in $\left|\widetilde{\mathcal{K}}_{m}\right|$ and extending linearly to the rest of the carrier. This is analogous to the Rodin and Sullivan construction of circle packing maps, but using the larger lattice structure rather than the individual triangles in the triangulation. 
In our earlier work [2, we showed that any torus covered by a circle packing can be deformed to any other torus by a sequence of refinements and weldings that keep the degree of the packings uniformly bounded. Equivalently, any lattice defined by a doubly periodic circle packing on the plane can be deformed into any other lattice by a sequence of refinements and weldings of each parallelogram of the lattice.

Thus given any constant Beltrami differential $\mu_{0}$, we can create both a linear map $f^{\mu_{0}}$ deforming the square lattice and a sequence of circle packings approximating the same deformation. Since our circle packing maps were defined by sending lattice points to lattice points and extending linearly, the circle packing maps $f_{m}$ converge uniformly to $f^{\mu_{0}}$.

Lemma 3.2. As $m \rightarrow \infty, f_{m}$ converges to $f^{\mu_{0}}$ uniformly on $\mathbb{C}$.

3.4. Nonconstant dilatations. If $\mu$ is not constant, we can still construct a sequence of circle packing maps converging to a quasiconformal map $f^{\mu}$ with dilatation $\mu$. Suppose $\mathcal{K}$ is again the triangulation of the ball-bearing packing $\mathcal{P}$ of Example 2.2 Since Beltrami differentials are measurable, we can find the average of $\mu$ over each square in the carrier $|K|$.

Now we treat $\mu$ as constant on each square and apply the refinement and welding procedure developed in the previous section. To approximate the effect of welding an infinite packing, first refine each square $n$ times, then refine and weld each of the new squares $m$ times. We thus produce packings $\widetilde{\mathcal{P}}_{n, m}$ and circle packing maps $f_{n, m}$.

Lemma 3.3. There exists a sequence $\left\{f_{k}\right\}$ of circle packing maps converging uniformly on compact subsets of $\mathbb{C}$ to a quasiconformal map whose dilatation on each square $Q$ of $\mathcal{K}$ is the average of $\mu$ over $Q$.

Proof. Fix a square $|Q|$ in $|\mathcal{K}|$ and let $\mu_{0}$ be the average of $\mu$ over $|Q|$. Let $\widetilde{\mathcal{Q}}_{n, m}$ be the portion of $\widetilde{\mathcal{P}}_{n, m}$ derived from $Q$. As $n \rightarrow \infty, \widetilde{\mathcal{Q}}_{n, m}$ must become indistinguishable from a scaled version of the infinite welded packing $\widetilde{\mathcal{P}}_{m}$ for $\mu_{0}$ constructed in Section 3.2 Otherwise, we could scale $\widetilde{\mathcal{Q}}_{n, m}$ to satisfy the same normalizations as the infinite packing. As $n \rightarrow \infty$, these would converge to a different infinite packing, thus violating uniqueness.

Consequently, for large $n, f_{n, m}$ must have approximately the same dilatation on compact subsets of $|Q|$ as the map $f_{m}$ constructed in the previous section using the infinite packings. Moreover, the boundaries between the original squares in $|K|$ have measure 0 . It follows via diagonalization that we can extract a sequence $\left\{f_{k}\right\}$ converging to a quasiconformal map with the required dilatation.

Now suppose we scale the original ball-bearing packing by $\epsilon$ (and relabel $\mathcal{C}_{1}$ ) before computing the average value of $\mu$ over each square. For each fixed $\epsilon$, Lemma 3.3 will still apply. Moreover, as $\epsilon \rightarrow 0$, the average values of $\mu$ on the tiny squares will converge to $\mu$. Thus by another diagonalization argument, we can extract a sequence of circle packing maps converging to $f^{\mu}$. Thus we have proved the following theorem.

Theorem 3.4. If $\mu$ is a Beltrami differential on $\mathbb{C}$ and $f^{\mu}$ is the corresponding normalized quasiconformal map, then our construction produces a sequence of circle packing maps converging to $f^{\mu}$. 


\section{Measurable Riemann mapping theorem}

4.1. Construction. Let $\Omega$ be a proper simply connected region in $\mathbb{C}$ and $\mu$ a Beltrami differential on $\Omega$. Let $z_{0}, z_{1} \in \Omega$. For each $n$, scale and translate the infinite ball-bearing packing of Example 2.2 so that the large "lattice" circles have radii less than $\frac{1}{n}$ and two circles $\mathcal{C}_{0}$ and $\mathcal{C}_{1}$ are centered at $z_{0}$ and $z_{1}$, respectively. Intersect this infinite packing with $\Omega$ and let $\mathcal{P}_{n}$ be the component containing $z_{0}$ and $z_{1}$. Let $\mathcal{K}_{n}$ denote the underlying triangulation of $\mathcal{P}_{n}$. For sufficiently large $n$, $\mathcal{P}_{n}$ will be nonempty, and the carrier $\left|K_{n}\right|$ will converge to $\Omega$ as $n \rightarrow \infty$.

Define $\mu_{n}$ on each square of $\left|\mathcal{K}_{n}\right|$ by setting it equal to the average value of $\mu$ over that square. Applying the same technique as in Section 3, we can construct a welding deformation $\widetilde{\mathcal{K}}_{n}$ of $\mathcal{K}_{n}$ which mirrors the quasiconformal deformation encoded in $\mu_{n}$. Since in this case $\widetilde{\mathcal{K}}_{n}$ is finite, it has a packing $\widetilde{\mathcal{P}}_{n}$ in $\mathbb{D}$. We then define $f_{n}:\left|\mathcal{K}_{n}\right| \rightarrow \mathbb{D}$ by sending centers of the lattice circles to centers of the corresponding circles in $\widetilde{\mathcal{P}}_{n}$ and extending linearly. We can assume $\widetilde{\mathcal{P}}_{n}$ is normalized so that $f_{n}\left(z_{0}\right)=0$ and $f_{n}\left(z_{1}\right)>0$.

4.2. Convergence. Using the same argument as Lemma 3.3. we see that increasing the number of refinements in the welding process causes $f_{n}$ to converge uniformly on compact subsets to the normalized quasiconformal map $f^{\mu_{n}}$ corresponding to $\mu_{n}$. Moreover, $\mu_{n} \rightarrow \mu$ and $f^{\mu_{n}} \rightarrow f^{\mu}$ as $n \rightarrow \infty$. Thus if we use sufficiently many refinements at each stage, we can ensure $f_{n}$ converges uniformly on compact subsets of $\Omega$ to $f^{\mu}$.

Circle Packing Measurable Riemann Mapping Theorem. If $\mu$ is a Beltrami differential on $\Omega$ and $f^{\mu}: \Omega \rightarrow \mathbb{D}$ is the corresponding normalized quasiconformal map, then our construction produces a sequence of circle packing maps converging to $f^{\mu}$.

Remark 4.1. One might reasonably ask whether our construction could be carried out for any packing in $\Omega$ instead of just the ball-bearing packing. Some loosening of this restriction seems possible, but at present, there is no method for distorting general packings in the way we can distort packings with a lattice structure.

\section{REFERENCES}

1. E. M. Andreev, Convex polyhedra of finite volume in Lobacevskii space, Math. USSR Sbornik 12 (1970), 255-259 (English). MR0273510 (42:8388)

2. R. W. Barnard and G. Brock Williams, Combinatorial excursions in moduli space, Pacific J. Math. 205 (2002), no. 1, 3-30. MR1921075 (2003f:52017)

3. Alan F. Beardon and Kenneth Stephenson, The uniformization theorem for circle packings, Indiana Univ. Math. J. 39 (1990), 1383-1425. MR1087197(92b:52038)

4. Christopher Bishop, Conformal welding and Koebe's theorem, preprint.

5. Philip L. Bowers and Kenneth Stephenson, A regular pentagonal tiling of the plane, Conform. Geom. Dyn. 1 (1997), 58-68. MR1479069 (99d:52016)

6. Tomasz Dubejko, Branched circle packings and discrete Blaschke products, Trans. Amer. Math. Soc. 347 (1995), no. 10, 4073-4103. MR.1308008 (95m:30045)

7. Tomasz Dubejko and Kenneth Stephenson, Circle packing: Experiments in discrete analytic function theory, Experiment. Math. 4 (1995), no. 4, 307-348. MR1387696 (97f:52027)

8. Frederick P. Gardiner and Nikola Lakic, Quasiconformal Teichmüller theory, Mathematical Surveys and Monographs, vol. 76, American Mathematical Society, 2000. MR.1730906 (2001d:32016)

9. Zheng-Xu He, Solving Beltrami equations by circle packing, Trans. Amer. Math. Soc. 322 (1990), 657-670. MR0974518 (91c:30032) 
10. Zheng-Xu He and Burt Rodin, Convergence of circle packings of finite valence to Riemann mappings, Comm. in Analysis and Geometry 1 (1993), 31-41. MR.1230272 (94m:30019)

11. Zheng-Xu He and Oded Schramm, Hyperbolic and parabolic packings, Discrete \& Computational Geom. 14 (1995), 123-149. MR1331923 (96h:52017)

12. - On the convergence of circle packings to the Riemann map, Invent. Math. 125 (1996), 285-305. MR1395721 (97i:30009)

13. P. Koebe, Kontaktprobleme der Konformen Abbildung, Ber. Sächs. Akad. Wiss. Leipzig, Math.-Phys. Kl. 88 (1936), 141-164.

14. O. Lehto, Univalent functions and Teichmüller spaces, Springer-Verlag, Berlin, Heidelberg, New York, 1987. MR0867407 (88f:30073)

15. O. Lehto and K.I. Virtanen, Quasiconformal mappings in the plane, second ed., SpringerVerlag, Berlin, Heidelberg, New York, 1973. MR0344463 (49:9202)

16. Al Marden and Burt Rodin, On Thurston's formulation and proof of Andreev's theorem, Computational Methods and Function Theory, Proceeding, Valparaiso 1989, Springer-Verlag, Berlin, Heidelberg, New York, Tokyo, 1990, Lecture Notes in Mathematics, Vol. 1435, pp. 103115. MR:1071766 (92b:52040)

17. Burt Rodin and Dennis Sullivan, The convergence of circle packings to the Riemann mapping, J. Differential Geometry 26 (1987), 349-360. MR0906396 (90c:30007)

18. Kenneth Stephenson, A probabilistic proof of Thurston's conjecture on circle packings, Rend. Sem. Mat. Fis. Milano 66 (1996), 201-291. MR1639851 (99m:52024)

19. William Thurston, The geometry and topology of 3-manifolds, Princeton University Notes, preprint.

20. sium at Purdue University on the occasion of the proof of the Bieberbach conjecture, March, 1985.

21. Masahiko Toki, Moduli of tori obtained by conformal sewing, preprint.

22. G. Brock Williams, Earthquakes and circle packings, J. Anal. Math. 85 (2001), 371-396. MR.1869616 (2003e:57032)

23. , Noncompact surfaces are packable, J. Anal. Math. 90 (2003). MR2001072 (2004h:30055)

24. —, Discrete conformal welding, Indiana Univ. Math. J. 53 (2004), no. 3, 765-804. MR2086700 (2005f:30018)

Department of Mathematics, Texas Tech University, Lubbock, Texas 79409

E-mail address: williams@math.ttu.edu

$U R L:$ http://www.math.ttu.edu/ williams 